\title{
ON THE SUCCESSIVE DERIVED SETS OF THE PISOT NUMBERS
}

\author{
DAVID W. BOYD
}

\begin{abstract}
Let $S$ denote the set of Pisot (or Pisot-Vijayaraghavan) numbers and let $S^{(k)}$ be the $k$ th derived set of $S$. It is shown that $k^{1 / 2}<\min S^{(k)}$ and that $\lim \sup \left(\min S^{(k)} / k\right)<1$. The lower bound improves the estimate $k^{1 / 4}<\min S^{(k)}$ of Dufresnoy and Pisot, while the upper bound improves the obvious estimate $\min S^{(k)}<k+1$.
\end{abstract}

The set $S$ of Pisot (or Pisot-Vijayaraghavan) numbers is the set of real algebraic integers $\theta>1$ all of whose remaining conjugates lie strictly within the unit circle. Salem [6] proved the remarkable fact that $S$ is a closed subset of the real line.

Let $S^{(k)}$ denote the $k$ th derived set of $S$, and $\min S^{(k)}$ its smallest element. It was shown by Dufresnoy and Pisot [2] that $\min S^{(k)}>k^{1 / 4}$, and it is known that the integer $k+1$ is in $S^{(k)}\left[5\right.$, p. 57] so $\min S^{(k)} \leqslant k+1$.

Our purpose here is to improve these results and to show that in fact $k^{1 / 2} \leqslant \min S^{(k)} \leqslant g(k)$ where $\lim \sup g(k) / k<1$. It is believed that the lower bound is closer to the truth.

THEOREM 1. $\min S^{(k)} \geqslant k^{1 / 2}$.

Proof. Let $C$ be the set of rational functions $A(z) / Q(z)=f(z)$ for which $A$ and $Q$ have integer coefficients, $Q(0)=1, Q$ has exactly one zero $\theta^{-1}$ in $|z| \leqslant 1$, and $|f(z)| \leqslant 1$ on $|z|=1$. Thus $\theta$ is in $S$, and $f$ is said to be associated with $\theta$. If $f(z)=u_{0}+u_{1} z+\ldots$, then it is shown in [3] that, for $n \geqslant 3$, there are polynomials $D_{n}, D_{n}^{*}$ of degree $n$ such that if

$$
E_{n}(z)=-z^{n} D_{n}\left(z^{-1}\right) \text { and } E_{n}^{*}(z)=z^{n} D_{n}^{*}\left(z^{-1}\right)
$$

then the Taylor expansions of $D_{n}(z) / E_{n}(z)$ and $D_{n}^{*}(z) / E_{n}^{*}(z)$ begin with the terms $u_{0}+\cdots+u_{n-1} z^{n-1}$, and furthermore, if the next terms are $w_{n} z^{n}$ and $w_{n}^{*} z^{n}$ respectively, then $w_{n} \leqslant u_{n} \leqslant w_{n}^{*}$. Also $D_{n}, D_{n}^{*}$ have exactly one zero each in $|z| \geqslant 1$ at the points $\tau_{n}, \tau_{n}^{*}$ where $\tau_{n} \leqslant \theta \leqslant \tau_{n}^{*}$ and $\tau_{n} \rightarrow \theta, \tau_{n}^{*} \rightarrow \theta$ as $n \rightarrow \infty$.

A result of Grandet-Hugot [4] states that if $\theta$ is in $S^{(k)}$ then there is an $f$ in

Received by the editors March 10, 1978.

AMS (MOS) subject classifications (1970). Primary 12A15; Secondary 30A42.

Key words and phrases. Pisot number, derived set. 
$C$ associated with $\theta$ such that

$$
w_{n}+k \leqslant u_{n} \leqslant w_{n}^{*}-k
$$

for all sufficiently large $n$.

Consider now the function

$$
\phi(z)=\left\{\left(D_{n}^{*} / E_{n}^{*}\right)-\left(D_{n} / E_{n}\right)\right\}\left(1-\tau_{n}^{*} z\right)\left(\tau_{n}^{*}-z\right)^{-1}\left(1-\tau_{n} z\right)\left(\tau_{n}-z\right)^{-1} z^{-n} \text {. }
$$

Clearly $\phi(z)$ is holomorphic in $|z|<1$, and $|\phi(z)|<2$ on $|z|=1$ since $\left|D_{n}^{*} / E_{n}^{*}\right|=1$ and $\left|D_{n} / E_{n}\right|=1$ on $|z|=1$. Thus

$$
\left(w_{n}^{*}-w_{n}\right) /\left(\tau_{n}^{*} \tau_{n}\right)=\phi(0) \leqslant 2 .
$$

By (1) and (2), $2 k \leqslant w_{n}^{*}-w_{n} \leqslant 2 \tau_{n}^{*} \tau_{n}$. Letting $n \rightarrow \infty$, we have $k \leqslant \theta^{2}$, which proves the theorem.

THEOREM 2. Let $\theta=n$, a rational integer. Then $n$ is in $S^{(k)}$, where

$$
k \geqslant \max (2[\alpha(n-1)], 2[\alpha(n-2)]+1),
$$

$\alpha$ being the constant $1 /(2 \sqrt{2}-1)>1 / 2$. In particular

$$
\lim \sup \left(\min S^{(k)} / k\right) \leqslant 1 /(2 \alpha)<1 .
$$

Proof. Let $P(a, b, c ; z)=z^{a}+z^{b}+z^{a+c}-z^{b+c}$ for integers $a, b, c$. Then

$$
|P(a, b, c ; z)|^{2}=\left|z^{a}+z^{b}\right|^{2}+\left(z^{c}+z^{-c}\right)\left(z^{a-b}-z^{b-a}\right)+\left|z^{a}-z^{b}\right|^{2} \leqslant 8,
$$

for $|z|=1$, since we can combine the first and last terms and use the parallelogram law. Thus, if $l-1 \geqslant 2 \sqrt{2} m$ and $n_{1}, \ldots, n_{3 m}$ are arbitrary positive integers, then the following polynomial has exactly one zero in $|z|<1$ :

$$
1-l z+P\left(n_{1}, n_{2}, n_{3} ; z\right)+\cdots+P\left(n_{3 m-2}, n_{3 m-1}, n_{3 m} ; z\right) .
$$

If we specialize $n_{3 i-2}=1$ for $1 \leqslant i \leqslant j$, where $j \leqslant m$, we obtain a polynomial with $3 m-j$ parameters whose root inside the unit circle tends to $1 /(l-j)$ as these parameters tend to $\infty$. This shows that $n=l-j \in S^{(3 m-j)}$ if $l-1>2 \sqrt{2} m$. The most favourable choice of $j$ is $j=m$, giving $n \in S^{(2 m)}$ if $n-1>(2 \sqrt{2}-1) m$, or else $j=m-1$, giving $n \in S^{(2 m+1)}$ if $n-2 \geqslant(2 \sqrt{2}-1) m$. These combine to prove the theorem.

REMARKS. 1. In [1], by a fairly lengthy analysis, we obtain an explicit formula for $\lim \left(w_{n}^{*}-w_{n}\right)=2 w(f)$, namely $w(f)=\theta^{2} \mathcal{G}\left(1-|f|^{2}\right)$, where $\mathcal{G}$ denotes the geometric mean on the unit circle. Using this in place of (2) one can improve the lower bound on $\min S^{(k)}$ to $\min S^{(k)} \geqslant(k+1)^{1 / 2}$.

2. It is known that $\min S=\theta_{0}=1.3247 \ldots$ the real root of $z^{3}-z-1$, that $\min S^{(1)}=\left(1+5^{1 / 2}\right) / 2$ [3], and $\min S^{(2)}=2$ [4]. No other explicit values of $\min S^{(k)}$ are known.

ACKNOWLEDGement. This work was supported in part by the Canadian National Research Council and a Senior Research Fellowship from the I. W. Killam Foundation. 


\section{REFERENCES}

1. D. W. Boyd, Pisot numbers and the width of meromorphic functions (privately circulated manuscript).

2. J. Dufresnoy and Ch. Pisot, Sur les dérivés successifs d'un ensemble fermé d'entiers algébriques, Ann. Sci. Ecole Norm. Sup. (3) 70 (1953), 105-133.

3. , Étude de certaines fonctions méromorphes bornées sur le cercle unité. Application à un emsemble fermé d'entiers algébriques, Ann. Sci. Ecole Norm. Sup. (3) 72 (1955), 69-92.

4. M. Grandet-Hugot, Ensembles fermés d'entiers algébriques, Ann. Sci. Ecole Norm. Sup. (3) 82 (1965), 1-35.

5. Ch. Pisot, Quelques aspects de la théorie des entiers algébriques, Séminaire de Mathématiques Supérieures, Université de Montréal, 1966.

6. R. Salem, A remarkable class of algebraic integers. Proof of a conjecture of Vijayaraghavan, Duke Math. J. 11 (1944), 103-108.

Department of Mathematics, The University of British Columbia, Vancouver, B.C., Canada V6T IW5 